\title{
H $^{2}$-KORN'S INEQUALITY AND THE NONCONFORMING ELEMENTS FOR THE STRAIN GRADIENT ELASTIC MODEL
}

\author{
HONGLIANG LI, PINGBING MING, AND HUIYU WANG
}

\begin{abstract}
We establish a new $\mathrm{H}^{2}$-Korn's inequality and its discrete analog, which greatly simplify the construction of nonconforming elements for a linear strain gradient elastic model. The Specht triangle 41 and the NZT tetrahedron 45 are analyzed as two typical representatives for robust nonconforming elements in the sense that the rate of convergence is independent of the small material parameter. We construct new regularized interpolation estimate and the enriching operator for both elements, and prove the error estimates under minimal smoothness assumption on the solution. Numerical results are consistent with the theoretical prediction.
\end{abstract}

\section{INTRODUCTION}

Let $u$ be the solution of the following boundary value problem:

$$
\left\{\begin{array}{rlrl}
\left(\iota^{2} \triangle-I\right)(\mu \triangle u+(\lambda+\mu) \nabla \nabla \cdot u) & =f & & \text { in } \Omega, \\
u=\partial_{n} u=0 & & \text { on } \partial \Omega,
\end{array}\right.
$$

where $\lambda$ and $\mu$ are the Lamé constants, and $\iota$ is the microscopic parameter satisfying $0<\iota \leq 1$. In particular, we are interested in the regime when $\iota$ is close to zero. This boundary value problem arises from a linear strain gradient elastic model proposed by Aifantis et al [4, 37], and $u$ is the displacement. This model may be regarded as a simplification of the more general strain gradient elasticity models 32 because it contains only one extra material parameter $\iota$ besides the Lamé constants $\lambda$ and $\mu$. This simplified strain gradient model successfully eliminated the strain singularity of the brittle crack tip field [19, and we refer to [18 and 21] for other strain gradient models.

The boundary value problem (1.1) is essentially a singularly perturbed elliptic system of fourth order due to the appearance of the strain gradient $\nabla \epsilon(u)$. $\mathrm{C}^{1}$ conforming finite elements such as Argyris triangle [5] seems a natural choice for discretization. The performance of Argyris triangle and several other $\mathrm{C}^{1}$-conforming finite elements has been carefully studied in 20, for a nonlinear strain gradient elastic model. A drawback of the $\mathrm{C}^{1}$-conforming elements is that the number of the

Date: April 20, 2021.

Key words and phrases. Strain gradient elasticity, $\mathrm{H}^{2}$-Korn's inequality, robust finite elements. 
degrees of freedom is large and high order polynomial has to be used in the shape functions, which is more pronounced for three dimensional problems; See, e.g., the finite element for a three-dimensional strain gradient model proposed in [36] locally has 192 degrees of freedom. We aim to develop some simple and robust nonconforming elements to avoid such difficulties. The robustness is understood in the sense that the elements converge uniformly in the energy norm with respect to $\iota$.

To this end, we firstly prove a new $\mathrm{H}^{2}$-Korn's inequality and its discrete analog in any dimension. This $\mathrm{H}^{2}$-Korn's inequality may be viewed as a quantitative version of the so-called vector version of J.L. Lions lemma [15, Theorem 6.19-1]; See also (2.12), while our proof is constructive and may be adapted to prove a Korn's inequality for piecewise $\mathrm{H}^{2}$ vector field (broken $\mathrm{H}^{2}-$ Korn's inequality for short), which may be viewed as a higher-order counterpart of BREnNER's seminal Korn's inequality [1] for piecewise $\mathrm{H}^{1}$ vector fields. Compared to the broken $\mathrm{H}^{2}$-inequality proved in [27, the jump term associated with the gradient tensor of the piecewise vector field may be dropped. Therefore, the degrees of freedom associated with the gradient tensor along each face or edge may be dropped, which simplify the construction of the elements. Based on this observation, all $\mathrm{H}^{1}$ conforming but $\mathrm{H}^{2}$ nonconforming elements are suitable for approximating this strain gradient model. We choose the Specht triangle [4] and the NZT tetrahedron [45] as two typical representatives. The Specht triangle is simpler than those in 27, because the elements therein locally belong to a 21 dimensional subspace of quintic polynomials, while the tensor products of the Specht triangle locally belong to an 18 dimensional subspace of quartic polynomials. It is worth mentioning that the broken $\mathrm{H}^{2}$-Korn's inequality may also be exploited to develop $\mathrm{C}^{0}$ interior penalty method [17, 12] for the strain gradient elastic model.

To prove the robustness of both elements, we construct a regularized interpolation operator and an enriching operator, and derive certain estimates for such operators, which are key to prove sharp error estimate for problems with less smooth solution. These two operators are also useful for strain gradient elasticity model with other type boundary conditions.

The remaining part of the paper is organized as follows. We prove the continuous and the broken $\mathrm{H}^{2}-$ Korn's inequalities in $\S 2$. The Specht triangle and the NTZ tetrahedron are introduced in $\S 3$ and the corresponding regularized interpolant are constructed and analyzed therein. We introduce enriching operators for both elements in $\S 4$, and derive the error bounds uniformly with respect to $\iota$ in the same part. The numerical tests of both elements are reported in the last Section, which confirm the theoretical prediction in $\S 4$.

Throughout this paper, the constant $C$ may differ from line to line, while it is independent of the mesh size $h$ and the materials parameter $\iota$. 


\section{2. $\mathrm{H}^{2}-$ KORN'S INEQUALITIES}

In this part we prove the $\mathrm{H}^{2}$-Korn's inequalities and the broken $\mathrm{H}^{2}$-Korn's inequalities. Let us fix some notations firstly.

2.1. Notations. Let $\Omega \subset \mathbb{R}^{d}$ be a bounded convex polytope. We shall use the standard notations for Sobolev spaces, norms and semi-norms [2]. The function space $L^{2}(\Omega)$ consists functions that are square integrable over $\Omega$, which is equipped with norm $\|\cdot\|_{L^{2}(\Omega)}$ and the inner product $(\cdot, \cdot)$. Let $H^{m}(\Omega)$ be the Sobolev space of square integrable functions whose weak derivatives up to order $m$ are also square integrable, the corresponding norm $\|v\|_{H^{m}(\Omega)}^{2}:=\sum_{k=0}^{m}|v|_{H^{k}(\Omega)}^{2}$ with the semi-norm $|v|_{H^{k}(\Omega)}^{2}:=\sum_{|\alpha|=k}\left\|\partial^{\alpha} v\right\|_{L^{2}(\Omega)}^{2}$.

For a positive number $s$ that is not an integer, $H^{s}(\Omega)$ is the fractional order Sobolev space. Let $m=\lfloor s\rfloor$ be the largest integer less than $s$ and $\varrho=s-m$. The sem-inorm $|v|_{H^{s}(\Omega)}$ and the norm $\|v\|_{H^{s}(\Omega)}$ are given by

$$
\begin{aligned}
& |v|_{H^{s}(\Omega)}^{2}=\sum_{|\alpha|=m} \int_{\Omega} \int_{\Omega} \frac{\left|\left(\partial^{\alpha}\right) v(x)-\left(\partial^{\alpha}\right) v(y)\right|^{2}}{|x-y|^{2+2 \varrho}} \mathrm{d} x \mathrm{~d} y, \\
& \|v\|_{H^{s}(\Omega)}^{2}=\|v\|_{H^{m}(\Omega)}^{2}+|v|_{H^{s}(\Omega)}^{2} .
\end{aligned}
$$

By [2, §7], the above definition for the fractional order Sobolev space $H^{s}(\Omega)$ is equivalent to the one obtained by interpolation, i.e.,

$$
H^{s}(\Omega)=\left[H^{m+1}(\Omega), H^{m}(\Omega)\right]_{\theta} \quad \text { with } \quad \theta=m+1-s .
$$

In particular, there exists $C$ that depends on $\Omega$ and $s$ such that

$$
\|v\|_{H^{s}(\Omega)} \leq C\|v\|_{H^{m+1}(\Omega)}^{1-\theta}\|v\|_{H^{m}(\Omega)}^{\theta} .
$$

For $s \geq 0, H_{0}^{s}(\Omega)$ is the closure in $H^{s}(\Omega)$ of the space of $C^{\infty}(\Omega)$ functions with compact supports in $\Omega$. In particular,

$$
\begin{aligned}
& H_{0}^{1}(\Omega):=\left\{v \in H^{1}(\Omega) \mid v=0 \text { on } \partial \Omega\right\}, \\
& H_{0}^{2}(\Omega):=\left\{v \in H^{2}(\Omega) \mid v=\partial_{n} v=0 \text { on } \partial \Omega\right\},
\end{aligned}
$$

where $\partial_{n} v$ is normal derivative of $v$.

For any vector-valued function $v$, its gradient $\nabla v$ is a matrix-valued function given by $(\nabla v)_{i j}=\partial_{i} v_{j}$ for $i, j=1, \cdots, d$. The strain tensor $\epsilon(v)$ is given by $\epsilon(v)=\frac{1}{2}\left(\nabla v+[\nabla v]^{T}\right)$ with $\epsilon_{i j}=\frac{1}{2}\left(\partial_{i} v_{j}+\partial_{j} v_{i}\right)$. The divergence operator is defined by $\nabla \cdot v=\sum_{i=1}^{d} \partial_{i} v_{i}$. The spaces $\left[H^{m}(\Omega)\right]^{d},\left[H_{0}^{m}(\Omega)\right]^{d}$ and $\left[L^{2}(\Omega)\right]^{d}$ are standard Sobolev spaces for the vector fields. Without abuse of notation, we employ $|\cdot|$ to denote the abstract value of a scalar, the $\ell_{2}$ norm of a vector, and the Euclidean norm of a matrix. Throughout this paper, we may drop the subscript $\Omega$ whenever no confusion occurs.

Let $\mathcal{T}_{h}$ be a simplicial triangulation of $\Omega$ with maximum mesh size $h$. We assume all elements in $\mathcal{T}_{h}$ are shape-regular in the sense of Ciarlet and Raviart [14, i.e., 
there exists a constant $\gamma$ such that $h_{K} / \rho_{K} \leq \gamma$, where $h_{K}$ is the diameter of element $K$, and $\rho_{K}$ is the diameter of the largest ball inscribed into $K$, and $\gamma$ is the so-called chunkiness parameter [13. We denote by $\mathcal{F}_{h}, \mathcal{E}_{h}$ and $\mathcal{V}_{h}$ the sets of $(d-1)$-dimensional faces, edges and vertices, respectively. Let $\mathcal{F}_{h}^{B}=\{f \in$ $\left.\mathcal{F}_{h} \mid f \subset \partial \Omega\right\}$ be the set of boundary faces. We denote by $\mathcal{F}_{h}^{I}=\mathcal{F}_{h} \backslash \mathcal{F}_{h}^{B}$ the set of interior faces. Similar notations apply to $\mathcal{E}_{h}$ and $\mathcal{V}_{h}$. We denote by $\mathcal{T}_{h}^{B}=\left\{K \in \mathcal{T}_{h} \mid \exists f \in \mathcal{F}_{h}^{B}\right.$, s.t. $\left.f \subset \partial K\right\}$ the set of boundary simplexes. Given a simplex or sub-simplex $B$, we let $\mathcal{T}_{h}(B)=\left\{K \in \mathcal{T}_{h} \mid B \subset K\right\}$ be the element star containing $B$. Similar notations apply to the set of faces, edges and vertices. For example, for any node $a \in \mathcal{V}_{h}^{B}$, the $\mathcal{E}_{h}^{B}(a)$ is the set of boundary edges sharing a common node $a$.

We classify the boundary vertices as follows. We say that a node $a \in \mathcal{V}_{h}^{B}$ is a flat node if the boundary edges set $\mathcal{E}_{h}^{B}(a)$ span a $(d-1)$-dimensional linear space. Otherwise, we say that $a$ is a sharp node. We let $\mathcal{V}_{h}^{B}=\mathcal{V}_{h}^{b} \cup \mathcal{V}_{h}^{\#}$, where $\mathcal{V}_{h}^{b}$ and $\mathcal{V}_{h}^{\#}$ denote the sets of the flat node and sharp node, respectively. For any sharp node $a \in \mathcal{V}_{h}^{\#}$, we may choose a set with $d$ linear independent boundary edges, i.e., $\mathcal{E}_{a}=\left\{e_{i} \in \mathcal{V}_{h}^{B}(a) \mid 1 \leq i \leq d\right\}$, such that $\mathcal{E}_{a}$ provide a basis of $\mathbb{R}^{d}$.

2.2. $\mathbf{H}^{2}$-Korn's inequality. We write the boundary value problem (1.1) into the following variational problem: Find $u \in\left[H_{0}^{2}(\Omega)\right]^{d}$ such that

$$
a(u, v)=(f, v) \quad \text { for all } \quad v \in\left[H_{0}^{2}(\Omega)\right]^{d},
$$

where the bilinear form $a$ is defined for any $v, w \in\left[H_{0}^{2}(\Omega)\right]^{d}$ as

$$
a(v, w):=(\mathbb{C} \epsilon(v), \epsilon(w))+\iota^{2}(\mathbb{D} \nabla \epsilon(v), \nabla \epsilon(w)),
$$

and the fourth-order tensors $\mathbb{C}$ and the sixth-order tensor $\mathbb{D}$ are defined by

$$
\mathbb{C}_{i j k l}=\lambda \delta_{i j} \delta_{k l}+2 \mu \delta_{i k} \delta_{j l} \quad \text { and } \quad \mathbb{D}_{i j k l m n}=\lambda \delta_{i l} \delta_{j k} \delta_{m n}+2 \mu \delta_{i l} \delta_{j m} \delta_{k n},
$$

respectively. Here $\delta_{i j}$ is the Kronecker delta function. The strain gradient $\nabla \epsilon(v)$ is a third order tensor defined by $(\nabla \epsilon(v))_{i j k}=\epsilon_{j k, i}$.

The wellposedness of Problem (2.2) depends on the coercivity of the bilinear form $a$ over $\left[H_{0}^{2}(\Omega)\right]^{d}$, which is a direct consequence of the following $\mathrm{H}^{2}-$ Korn's inequality:

$$
\|\epsilon(v)\|_{L^{2}}^{2}+\|\nabla \epsilon(v)\|_{L^{2}}^{2} \geq C(\Omega)\|\nabla v\|_{H^{1}}^{2} \quad \text { for all } \quad v \in\left[H_{0}^{2}(\Omega)\right]^{d} .
$$

This inequality was proved in [27, Theorem 1$]$ by exploiting the community property of the strain operator $\epsilon$ and the partial derivative operator $\partial$, which has been implicitly used in [1] to prove an inequality similar to (2.3).

In what follows, we prove that (2.3) remains valid for a more general vector field $v$ in $\left[H^{2}(\Omega) \cap H_{0}^{1}(\Omega)\right]^{d}$. The precise form is stated in (2.4). Our proof relies on the fact that the strain gradient field fully controls the Hessian of the displacement algebraically; See, cf. (2.5). This fact will be further exploited to prove a discrete 
analog of (2.4) for a piecewise vector field, which is dubbed as the broken $\mathrm{H}^{2}-$ Korn's inequality. We have exploited a weaker version of such broken $\mathrm{H}^{2}-$ Korn's inequality to design two robust strain gradient finite elements in [27.

Theorem 2.1. For any $v \in\left[H_{0}^{1}(\Omega)\right]^{d}$ and $\nabla \epsilon(v) \in\left[L^{2}(\Omega)\right]^{d \times d \times d}$, there holds $\nabla v \in\left[H^{1}(\Omega)\right]^{d \times d}$ and

$$
\|\epsilon(v)\|_{L^{2}}^{2}+\|\nabla \epsilon(v)\|_{L^{2}}^{2} \geq \frac{1}{4}\left(\|\nabla v\|_{L^{2}}^{2}+\left\|D^{2} v\right\|_{L^{2}}^{2}\right) .
$$

Here, the constant $1 / 4$ in right hand side of the above inequality may be replaced by $1-1 / \sqrt{2}$ when $d=2$.

Proof. The core of the proof is the following algebraic inequality:

$$
|\nabla \epsilon(v)|^{2} \geq \frac{1}{4}\left|\nabla^{2} v\right|^{2}
$$

Integrating (2.5) over domain $\Omega$, we obtain

$$
\|\nabla \epsilon(v)\|_{L^{2}}^{2} \geq \frac{1}{4}\left\|\nabla^{2} v\right\|_{L^{2}}^{2},
$$

which together with the first Korn's inequality [25, 26]:

$$
2\|\epsilon(v)\|_{L^{2}}^{2} \geq\|\nabla v\|_{L^{2}}^{2} \quad \text { for all } \quad v \in\left[H_{0}^{1}(\Omega)\right]^{d}
$$

implies (2.4).

To prove (2.5), we start with the identity

$$
\begin{aligned}
|\nabla \epsilon(v)|^{2}= & \sum_{1 \leq i, j, k \leq d}\left|\partial_{i} \epsilon_{j k}\right|^{2} \\
= & \sum_{1=1}^{d}\left|\partial_{i} \epsilon_{i i}\right|^{2}+\sum_{1 \leq i<j \leq d}\left(\left|\partial_{i} \epsilon_{j j}\right|^{2}+2\left|\partial_{j} \epsilon_{i j}\right|^{2}\right)+\left(\left|\partial_{j} \epsilon_{i i}\right|^{2}+2\left|\partial_{i} \epsilon_{i j}\right|^{2}\right) \\
& +\sum_{i \neq j \neq k}\left|\partial_{i} \epsilon_{j k}\right|^{2}:=I_{1}+I_{2}+I_{3},
\end{aligned}
$$

where $I_{3}$ vanishes for $d=2$.

Employing the elementary algebraic inequality

$$
a^{2}+\frac{1}{2}(a+b)^{2} \geq(1-1 / \sqrt{2})\left(a^{2}+b^{2}\right), \quad a, b \in \mathbb{R},
$$

we obtain

$$
\left\{\begin{array}{l}
\left|\partial_{j} \epsilon_{i i}\right|^{2}+2\left|\partial_{i} \epsilon_{i j}\right|^{2} \geq(1-1 / \sqrt{2})\left(\left|\partial_{i j} v_{i}\right|^{2}+\left|\partial_{i i} v_{j}\right|^{2}\right) \\
\left|\partial_{i} \epsilon_{j j}\right|^{2}+2\left|\partial_{j} \epsilon_{i j}\right|^{2} \geq(1-1 / \sqrt{2})\left(\left|\partial_{i j} v_{j}\right|^{2}+\left|\partial_{j j} v_{i}\right|^{2}\right) .
\end{array}\right.
$$

Using the elementary algebraic equality

$$
\sum_{i=1}^{d}\left(a_{i}+a_{i+1}\right)^{2}=\sum_{i=1}^{d} a_{i}^{2}+\left(\sum_{i=1}^{d} a_{i}\right)^{2} \quad \text { for } \quad a_{i} \in \mathbb{R} \quad \text { and } \quad a_{d+1}=a_{1},
$$


we obtain

$$
\sum_{1 \leq i \neq j \neq k \leq d}\left|\partial_{i} \epsilon_{j k}\right|^{2}=\frac{1}{4} \sum_{1 \leq i \neq j \neq k \leq d}\left|\partial_{i j} v_{k}\right|^{2}+\frac{1}{4}\left(\sum_{1 \leq i \neq j \neq k \leq d} \partial_{i j} v_{k}\right)^{2} .
$$

Combining (2.8), (2.9) and (2.10), we obtain (2.5) immediately.

$I_{3}$ vanishes when $d=2$. Therefore, the constant in the right-hand side of (2.4) may be replaced by $1-1 / \sqrt{2}$. This completes the proof.

A direct consequence of Theorem 2.1 is the following full $\mathrm{H}^{2}$-Korn's inequality.

Corollary 2.2. Let $\Omega \subset \mathbb{R}^{d}$ be a domain such that the following Korn's inequality is valid for any vector field $v \in\left[L^{2}(\Omega)\right]^{d}$ and $\epsilon(v) \in\left[L^{2}(\Omega)\right]^{d \times d}$,

$$
\|v\|_{L^{2}}+\|\epsilon(v)\|_{L^{2}} \geq C(\Omega)\|v\|_{H^{1}} .
$$

If $v \in\left[L^{2}(\Omega)\right]^{d}, \epsilon(v) \in\left[L^{2}(\Omega)\right]^{d \times d}$ and $\nabla \epsilon(v) \in\left[L^{2}(\Omega)\right]^{d \times d \times d}$, then $v \in\left[H^{2}(\Omega)\right]^{d}$ and

$$
\|v\|_{L^{2}}+\|\epsilon(v)\|_{L^{2}}+\|\nabla \epsilon(v)\|_{L^{2}} \geq \min (C(\Omega), 1 / 2)\|v\|_{H^{2}} .
$$

In [15, Theorem 6.19-1], the following vector version of J.L. Lions Lemma is proved: For any domain $D$ in $\mathbb{R}^{d}$ and $m \in \mathbb{Z}$, then

$$
v \in\left[H^{m}(D)\right]^{d} \text { and } \quad \epsilon(v) \in\left[H^{m}(D)\right]^{d \times d} \text { implies } v \in\left[H^{m+1}(D)\right]^{d} .
$$

The inequality (2.11) may be viewed as a quantitative version of (2.12) with $m=1$, while the proof in [15, Theorem 6.19-1] is nonconstructive and is not easy to be adapted to prove the broken $\mathrm{H}^{2}$-Korn's inequality for a piecewise vector filed.

The regularity of Problem (2.2) is essential to prove a uniform error estimate. Unfortunately, it does not seem easy to identify such estimates in the literature, and we give a proof for the readers' convenience. We firstly make an extra regularity assumption.

Hypothesis 2.3. Let $u$ be a solution of following equations,

$$
\begin{cases}\triangle(\mathcal{L} u)=f & \text { in } \Omega, \\ u=\partial_{n} u=0 & \text { on } \partial \Omega,\end{cases}
$$

where $\mathcal{L} u:=\mu \triangle u+(\lambda+\mu) \nabla \nabla \cdot u$. Then there holds that for any $f \in H^{-1}(\Omega)$,

$$
\|u\|_{H^{3}} \leq C\|f\|_{H^{-1}} .
$$

If $\Omega$ is a smooth domain, the regularity property (2.13) is standard; See e.g., 3 . While it is unclear whether the above regularity estimate is true for a convex polytope. Nevertheless, if the operator $\mathcal{L}$ is replaced by the Laplacian operator, then (2.13) is proved in [31, Chapter 4 Theorem 4.3.10]. 
Lemma 2.4. Assume Hypothesis 2.3 is valid and let $u$ be the solution of (2.2), then there exists $C$ that may depend on $\Omega$ but independent of $\iota$ such that

$$
\left\|\nabla^{k}\left(u-u_{0}\right)\right\|_{L^{2}} \leq C \iota^{3 / 2-k}\|f\|_{L^{2}} \quad \text { for } \quad k=1,2,
$$

where $u_{0} \in\left[H_{0}^{1}(\Omega)\right]^{d}$ satisfies

$$
\left(\mathbb{C} \epsilon\left(u_{0}\right), \epsilon(v)\right)=(f, v) \quad \text { for all } \quad v \in\left[H_{0}^{1}(\Omega)\right]^{d} .
$$

Moreover, we have

$$
\|u\|_{H^{3 / 2}} \leq C\|f\|_{L^{2}}
$$

and

$$
\|u\|_{H^{5 / 2}} \leq C \iota^{-1}\|f\|_{L^{2}} .
$$

Under Hypothesis 2.3. we may prove this regularity result by following essentially the same line of the proof in [35, Lemma 5.1]. We include it here for completeness.

Proof. By (1.1) and (2.15), we have

$$
\triangle \mathcal{L}(u)=\iota^{-2} \mathcal{L}\left(u-u_{0}\right) .
$$

Using the regularity hypothesis (2.13), we obtain

$$
\|u\|_{H^{3}} \leq C \iota^{-2}\left\|\mathcal{L}\left(u-u_{0}\right)\right\|_{-1} \leq C \iota^{-2}\left(\mathbb{C} \epsilon\left(u-u_{0}\right), \epsilon\left(u-u_{0}\right)\right)^{\frac{1}{2}} .
$$

By the standard regularity estimate, we have

$$
\left\|u_{0}\right\|_{H^{2}} \leq C\|f\|_{L^{2}} .
$$

Denoting $\phi=u-u_{0}$ and integration by parts, we have

$$
\begin{aligned}
\iota^{2}(\mathbb{D} \nabla \epsilon(\phi), \nabla \epsilon(\phi))+(\mathbb{C} \epsilon(\phi), \epsilon(\phi))= & -\iota^{2}\left(\mathbb{D} \nabla \epsilon\left(u_{0}\right), \nabla \epsilon(\phi)\right) \\
& +\iota^{2} \int_{\partial \Omega} M_{n n}(u) \partial_{n} u_{0} \mathrm{~d} \sigma(x),
\end{aligned}
$$

where $M_{n n}(u)=n^{\mathrm{T}} \cdot \mathbb{D} \nabla \epsilon(u) \cdot n$.

Using the regularity estimate (2.19), we obtain

$$
\iota^{2}\left(\mathbb{D} \nabla \epsilon\left(u_{0}\right), \nabla \epsilon(\phi)\right) \leq \frac{\iota^{2}}{2}(\mathbb{D} \nabla \epsilon(\phi), \nabla \epsilon(\phi))+\frac{\iota^{2}}{2}\|f\|_{L^{2}}^{2} .
$$

Using the trace inequality (2.24), we obtain, for $\delta>0$ to be chosen later,

$$
\begin{aligned}
\iota^{2}\left|\int_{\partial \Omega} M_{n n}(u) \partial_{n} u_{0} \mathrm{~d} \sigma(x)\right| & \leq \iota^{3} \delta\left\|M_{n n}(u)\right\|_{L^{2}(\partial \Omega)}^{2}+\frac{\iota}{4 \delta}\left\|\partial_{n} u_{0}\right\|_{L^{2}(\partial \Omega)}^{2} \\
& \leq C \delta\left(\iota^{4}\left\|\nabla^{2} u\right\|_{H^{1}}^{2}+\iota^{2}\left\|\nabla^{2} u\right\|_{L^{2}}^{2}\right)+\frac{C}{\delta} \iota\left\|u_{0}\right\|_{H^{2}}^{2} .
\end{aligned}
$$

Using (2.6), we obtain

$$
\left\|\nabla^{2} u\right\|_{L^{2}}^{2} \leq 2\left\|\nabla^{2} \phi\right\|_{L^{2}}^{2}+2\left\|\nabla^{2} u_{0}\right\|_{L^{2}}^{2} \leq \frac{4}{\mu}(\mathbb{D} \nabla \epsilon(\phi), \nabla \epsilon(\phi))+2\left\|\nabla^{2} u_{0}\right\|_{L^{2}}^{2} .
$$


Using the regularity estimates (2.18) and (2.19), we bound the right-hand side of the above inequality as

$$
\begin{aligned}
\iota^{2}\left|\int_{\partial \Omega} M_{n n}(u) \partial_{n} u_{0} \mathrm{~d} \sigma(x)\right| \leq & C \delta\left[(\mathbb{C} \epsilon(\phi), \epsilon(\phi))+\iota^{2}(\mathbb{D} \nabla \epsilon(\phi), \nabla \epsilon(\phi))\right] \\
& +C \iota\left(\delta \iota+\frac{1}{\delta}\right)\|f\|_{L^{2}}^{2} .
\end{aligned}
$$

Combining the above inequalities, we obtain

$$
\begin{aligned}
\iota^{2}(\mathbb{D} \nabla \epsilon(\phi), \nabla \epsilon(\phi))+(\mathbb{C} \epsilon(\phi), \epsilon(\phi)) \leq & C \delta\left[(\mathbb{C} \epsilon(\phi), \epsilon(\phi))+\iota^{2}(\mathbb{D} \nabla \epsilon(\phi), \nabla \epsilon(\phi))\right] \\
& +\frac{\iota^{2}}{2}(\mathbb{D} \nabla \epsilon(\phi), \nabla \epsilon(\phi))+C \iota^{2} \delta\|f\|_{L^{2}}^{2}+\frac{C \iota}{\delta}\|f\|_{L^{2}}^{2} .
\end{aligned}
$$

Choosing $\delta$ properly, we obtain (2.14).

Using (2.14) and Poincaré inequality, and noting that $\iota<1$, we obtain

$$
\left\|u-u_{0}\right\|_{H^{2}} \leq C\left(\iota^{1 / 2}+\iota^{-1 / 2}\right)\|f\|_{L^{2}} \leq C \iota^{-1 / 2}\|f\|_{L^{2}}
$$

and

$$
\|u\|_{H^{2}} \leq\left\|u-u_{0}\right\|_{H^{2}}+\left\|u_{0}\right\|_{H^{2}} \leq C \iota^{-1 / 2}\|f\|_{L^{2}} .
$$

Interpolating (2.20) and (2.14) with $k=1$, we obtain

$$
\left\|u-u_{0}\right\|_{H^{3 / 2}} \leq C\|f\|_{L^{2}} .
$$

Using the interpolation inequality (2.1), we obtain

$$
\left\|u_{0}\right\|_{H^{3 / 2}} \leq C\left\|u_{0}\right\|_{H^{1}}^{1 / 2}\left\|u_{0}\right\|_{H^{2}}^{1 / 2} \leq C\|f\|_{L^{2}} .
$$

A combination of the above two inequalities yields (2.16).

Combining (2.18) and (2.14), we obtain

$$
\|u\|_{H^{3}} \leq C \iota^{-2}\left\|\nabla\left(u-u_{0}\right)\right\|_{L^{2}} \leq C \iota^{-3 / 2}\|f\|_{L^{2}} .
$$

Interpolating the above inequality and (2.21), we obtain (2.17).

2.3. The broken $\mathbf{H}^{2}$-Korn's inequality. For any $m \in \mathbb{N}$, the space of piecewise vector fields is defined by

$$
\left[H^{m}\left(\Omega, \mathcal{T}_{h}\right)\right]^{d}:=\left\{v \in\left[L^{2}(\Omega)\right]^{d}|v|_{K} \in\left[H^{m}(K)\right]^{d} \quad \text { for all } \quad K \in \mathcal{T}_{h}\right\},
$$

which is equipped with the broken norm

$$
\|v\|_{H_{h}^{k}}:=\|v\|_{L^{2}}+\sum_{k=1}^{m}\left\|\nabla_{h}^{k} v\right\|_{L^{2}},
$$

where $\left\|\nabla_{h}^{k} v\right\|_{L^{2}}^{2}=\sum_{K \in \mathcal{T}_{h}}\left\|\nabla^{k} v\right\|_{L^{2}(K)}^{2}$ with $\left.\left(\nabla_{h}^{k} v\right)\right|_{K}=\nabla^{k}\left(\left.v\right|_{K}\right)$. Moreover, $\epsilon_{h}(v)=\left(\nabla_{h} v+\left[\nabla_{h} v\right]^{T}\right) / 2$. For any $v \in H^{m}\left(\Omega, \mathcal{T}_{h}\right)$, we denote by $\llbracket v \rrbracket$ the jump of $v$ across the faces or the edge.

The main result of this part is the following broken $\mathrm{H}^{2}$-Korn's inequality. 
Theorem 2.5. For any $v \in\left[H^{2}\left(\Omega, \mathcal{T}_{h}\right)\right]^{d}$, there exits $C$ that depends on $\Omega$ and $\gamma$ but independent of $h$ such that

$$
\begin{aligned}
\|v\|_{H_{h}^{2}}^{2} \leq C\left(\left\|\nabla_{h} \epsilon_{h}(v)\right\|_{L^{2}}^{2}+\right. & \left\|\epsilon_{h}(v)\right\|_{L^{2}}^{2}+\|v\|_{L^{2}}^{2} \\
& \left.+\sum_{f \in \mathcal{F}_{h}} h_{e}^{-1}\left\|\llbracket \Pi_{f} v \rrbracket\right\|_{L^{2}(f)}^{2}\right),
\end{aligned}
$$

where $\Pi_{f}:\left[L^{2}(f)\right]^{d} \mapsto\left[P_{1,-}(f)\right]^{d}$ is the $L^{2}$ projection and

$$
\left[P_{1,-}(f)\right]^{d}:=\left\{v \in\left[P_{1}(f)\right]^{d} \mid v \cdot t \in R M(F)\right\},
$$

where $t$ is the tangential vector of the face $f$ (or edge for $d=2$ ), and $R M(f)$ is the infinitesimal rigid motion on $f$.

For a piecewise vector filed $v$, the inequality (2.22) improves the one proved in [27, Theorem 2] by removing the jump term

$$
\sum_{i=1}^{2} \sum_{f \in \mathcal{F}_{h}} h_{f}^{-1}\left\|\llbracket \Pi_{f}\left(v_{, i}\right) \rrbracket\right\|_{L^{2}(f)}^{2} .
$$

This term stands for the jump of the gradient tensor of the vector field $v$ across the element boundary. This would simplify the construction of the robust strain gradient elements as shown in the next two parts.

Proof of Theorem 2.5 Integrating (2.5) over element $K \in \mathcal{T}_{h}$, we obtain,

$$
\|\nabla \epsilon(v)\|_{L^{2}(K)}^{2} \geq \frac{1}{4}\left\|\nabla^{2} v\right\|_{L^{2}(K)}^{2} .
$$

Summing up all $K \in \mathcal{T}_{h}$, we get

$$
\left\|\nabla_{h} \epsilon_{h}(v)\right\|_{L^{2}} \geq \frac{1}{4}\left\|\nabla_{h}^{2} v\right\|_{L^{2}}^{2},
$$

which together with the following Korn's inequality for a piecewise $\mathrm{H}^{1}$ vector filed proved by Mardal and Winther [30]

$$
\|v\|_{H_{h}^{1}}^{2} \leq C\left(\left\|\epsilon_{h}(v)\right\|_{L^{2}}^{2}+\|v\|_{L^{2}}^{2}+\sum_{f \in \mathcal{F}_{h}} h_{f}^{-1}\left\|\llbracket \Pi_{f} v \rrbracket\right\|_{L^{2}(f)}^{2}\right)
$$

implies (2.22).

We shall frequently use the following trace inequalities.

Lemma 2.6. For any Lipschitz domain $D$, there exists $C$ depending on $D$ such that

$$
\|v\|_{L^{2}(\partial D)} \leq C\|v\|_{L^{2}(D)}^{1 / 2}\|v\|_{H^{1}(D)}^{1 / 2} .
$$

For an element $K$, there exists $C$ independent of $h_{K}$, but depends on $\gamma$ such that

$$
\|v\|_{L^{2}(\partial K)} \leq C\left(h_{K}^{-1 / 2}\|v\|_{L^{2}(K)}+h_{K}^{1 / 2}\|\nabla v\|_{L^{2}(K)}\right) .
$$


If $v \in \mathbb{P}_{m}(K)$, then there exists $C$ independent of $v$, but depends on $\gamma$ and $m$ such that

$$
\|v\|_{L^{2}(\partial K)} \leq C h_{K}^{-1 / 2}\|v\|_{L^{2}(K)} .
$$

The multiplicative type trace inequality (2.24) may be found in 23 , Theorem 1.5.1.10], while (2.25) is a direct consequence of (2.24). The third trace inequality is a combination of (2.25) and the inverse inequality for any polynomial $v \in \mathbb{P}_{m}(K)$.

\section{INTERPOLATION FOR NONSMOOTH DATA}

Motivated by the broken $\mathrm{H}^{2}$-Korn's inequality (2.22), we conclude that the $\mathrm{H}^{1}$-conforming but $\mathrm{H}^{2}$-nonconforming finite elements are natural choices for approximating Problem (3.2). A family of rectangular elements in this vein may be found in 29, and two nonconforming tetrahedron elements were constructed and analyzed in [44. Note that the tensor product of certain finite elements for the singular perturbation problem of fourth order may also be used to approximate (1.1), we refer to 39, 40, 24, 35, 42 and references therein for such elements. In what follows, we select the Specht triangle [41] and the NZT tetrahedron [45] as the representatives. The Specht triangle is a successful plate bending element, which passes all the patch tests and performs excellently, and is one of the best thin plate triangles with 9 degrees of freedom that currently available [48, Quatation in p. 345]. The NZT tetrahedron may be regarded as a three-dimensional extension of the Specht triangle.

The Specht triangle and the NZT tetrahedron may be defined by the finite element triple $\left(K, P_{K}, \Sigma_{K}\right)$ [14 in a unifying way as following: $K$ is a simplex, and

$$
\left\{\begin{array}{l}
P_{K}=Z_{K}+b_{K} \mathbb{P}_{1}(K) \\
\Sigma_{K}=\left\{p\left(a_{i}\right),\left(e_{i j} \cdot \nabla p\right)\left(a_{i}\right), 1 \leq i \neq j \leq d+1\right\}
\end{array}\right.
$$

with extra constraints

$$
\frac{1}{\left|f_{i}\right|} \int_{f_{i}} \partial_{n} p=\frac{1}{d} \sum_{1 \leq k \leq d+1, k \neq i} \partial_{n} p\left(a_{k}\right), \quad i=1, \cdots, d+1,
$$

where $f_{i}$ is a $(d-1)$ dimensional simplex opposite to vertex $a_{i}$, and $e_{i j}$ is the edge vector from $a_{i}$ to $a_{j}$. Here $Z_{K}$ is the Zienkiewicz space defined by

$$
Z_{K}=\mathbb{P}_{2}(K)+\operatorname{Span}\left\{\lambda_{i}^{2} \lambda_{j}-\lambda_{i} \lambda_{j}^{2} \mid 1 \leq i \neq j \leq d+1\right\}
$$

where $\lambda_{i}$ is the barycentric coordinates associated with the vertex $a_{i}$.

The finite element space is define by

$$
X_{h}:=\left\{v \in H^{1}(\Omega)|v|_{K} \in P_{K}, K \in \mathcal{T}_{h} ; v(a), \nabla v(a) \text { are continuous for } a \in \mathcal{V}_{h}\right\} .
$$

The corresponding homogenous finite element space is defined by

$$
X_{h}^{0}:=\left\{v \in X_{h} \mid v(a), \nabla v(a) \text { vanish on } \partial \Omega \text { for } a \in \mathcal{V}_{h}\right\} .
$$


It is clear that $X_{h}^{0} \subset H_{0}^{1}(\Omega)$. We denote $V_{h}=\left[X_{h}^{0}\right]^{d}$, and approximating problem reads as: Find $u_{h} \in V_{h}$ such that

$$
a_{h}\left(u_{h}, v\right)=(f, v) \text { for all } v \in V_{h},
$$

where the bilinear form $a_{h}$ is defined for any $v, w \in V_{h}$ as

$$
a_{h}(v, w):=(\mathbb{C} \epsilon(v), \epsilon(w))+\iota^{2}\left(\mathbb{D} \nabla_{h} \epsilon(v), \nabla_{h} \epsilon(w)\right)
$$

with

$$
\left(\mathbb{D} \nabla_{h} \epsilon(v), \nabla_{h} \epsilon(w)\right):=\sum_{K \in \mathcal{T}_{h}} \int_{K} \mathbb{D} \nabla \epsilon(v) \nabla \epsilon(w) \mathrm{d} x .
$$

The energy norm is defined as $\|v\|_{\iota, h}:=\left(\|v\|_{H^{1}}^{2}+\iota^{2}\left\|\nabla_{h}^{2} v\right\|_{L^{2}}^{2}\right)^{1 / 2}$. The bilinear form is coercive in this energy norm as shown in the next lemma.

Lemma 3.1. For any $v \in V_{h}$,

$$
a_{h}(v, v) \geq \frac{\mu}{2+2\left|C_{p}\right|^{2}}\|v\|_{\iota, h}^{2},
$$

where $C_{p}$ appears in the Poincaré inequality

$$
\|v\|_{L^{2}} \leq C_{p}\|\nabla v\|_{L^{2}} \quad \text { for all } \quad v \in\left[H_{0}^{1}(\Omega)\right]^{d} .
$$

The estimate (3.3) immediately implies the wellposedness of Problem (3.2) for any fixed $\iota$.

Proof. For any $v \in V_{h}$, there holds

$$
a_{h}(v, v) \geq 2 \mu\left(\|\epsilon(v)\|_{L^{2}}^{2}+\iota^{2}\left\|\nabla_{h} \epsilon(v)\right\|_{L^{2}}^{2}\right) .
$$

Using the first Korn's inequality (2.7) and the estimate (2.23), we obtain

$$
a_{h}(v, v) \geq \frac{\mu}{2}\left(\|\nabla v\|_{L^{2}}^{2}+\iota^{2}\left\|\nabla_{h}^{2} v\right\|_{L^{2}}^{2}\right) .
$$

The coercivity estimate (3.3) follows by using the Poincare's inequality (3.4).

The standard interpolation estimate for the above elements reads as [16],

$$
\sum_{j=0}^{3} h^{j}\left\|\nabla_{h}^{j}(v-\Pi v)\right\|_{L^{2}} \leq C h^{3}\left\|\nabla^{3} v\right\|_{L^{2}},
$$

This interpolant is unbounded in $H^{2}(\Omega)$, which is even not well-defined for a function in $H^{2}(\Omega)$.

Our definition for the regularized interpolant is a combination of a regularized interpolant in 24] and an enriching operator defined in [34.

Define $I_{h}:=\Pi_{h} \circ \Pi_{C}$ with $\Pi_{C}: H_{0}^{1}(\Omega) \rightarrow L_{h}$ the Scott-Zhang interpolant [38, where $L_{h}$ is the quadratic Lagrangian finite element space with vanishing trace. The operator $\Pi_{h}: L_{h} \rightarrow X_{h}$ is locally defined as follows.

(1) If $a \in \mathcal{V}_{h}^{I}$ is an interior vertex, then we fix an element $K_{a}$ from $\mathcal{T}_{h}(a)$,

$$
\Pi_{h} w(a):=w(a) \quad \text { and } \quad \nabla \Pi_{h} w(a):=\nabla w_{\left.\right|_{K_{a}}}(a) .
$$


(2) If $a \in \mathcal{V}_{h}^{b}$ is a flat node, then we fix an element $K_{a}$ from $\mathcal{T}_{h}{ }^{B}(a)$,

$$
\Pi_{h} w(a):=0 \quad \text { and } \quad \nabla \Pi_{h} w(a):=\nabla w_{\left.\right|_{K_{a}}}(a) .
$$

(3) If $a \in \mathcal{V}_{h}^{\#}$ is a sharp node, then

$$
\Pi_{h} w(a):=0 \quad \text { and } \quad \nabla \Pi_{h} w(a):=0 .
$$

Remark 3.2. For any $w \in H_{0}^{1}(\Omega) \cap L_{h}$, there holds $\Pi_{h} w \in H_{0}^{1}(\Omega)$.

For any $v \in H_{0}^{2}(\Omega)$, we define $I_{h}^{0}: H_{0}^{2}(\Omega) \rightarrow X_{h}^{0}$ with

$$
I_{h}^{0} v(a)=I_{h} v(a), \nabla I_{h}^{0} v(a)=\nabla I_{h} v(a), \text { for all } a \in \mathcal{V}_{h}^{I}
$$

Theorem 3.3. There exists an operator $I_{h}: H_{0}^{1}(\Omega) \rightarrow X_{h}$ such that for any $v \in H^{m}(\Omega)$ with $2 \leq m \leq 3$, there holds

$$
\left\|v-I_{h} v\right\|_{H_{h}^{k}} \leq C h^{m-k}|v|_{H^{m}}, \quad 0 \leq k \leq m .
$$

Moreover, there exists $I_{h}^{0}: H_{0}^{2}(\Omega) \rightarrow X_{h}^{0}$ such that for any $v \in H^{m}(\Omega) \cap H_{0}^{2}(\Omega)$ with $2 \leq m \leq 3$, there holds

$$
\left\|v-I_{h}^{0} v\right\|_{H_{h}^{k}} \leq C h^{m-k}|v|_{H^{m}}, \quad 0 \leq k \leq m .
$$

The interpolant $I_{h}^{0} v$ is enough to our ends, while the more general interpolant $I_{h} v$ is a useful tool to deal with the strain gradient model with other boundary conditions; See, e.g., [6].

Proof. For any $\phi \in P_{K}$, a standard scaling argument yields that

$$
\|\phi\|_{L^{2}(K)}^{2} \leq C h_{K}^{d} \sum_{a \in \mathcal{V}_{K}}\left(|\phi(a)|^{2}+h_{K}^{2}|\nabla \phi(a)|^{2}\right),
$$

where $\mathcal{V}_{K}$ is the set containing all vertices of $K$. Let $w=\Pi_{C} v$ and $\phi=w-\Pi_{h} w$. Noting $\phi(a)=0$, we obtain

$$
\|\phi\|_{L^{2}(K)}^{2} \leq C h_{K}^{d+2} \sum_{a \in \mathcal{V}_{K}}|\nabla \phi(a)|^{2} .
$$

If the node $a \in \mathcal{V}_{h}^{I}$ or $a \in \mathcal{V}_{h}^{b}$, there has $\nabla \Pi_{h} w(a)=\left(\nabla w_{\left.\right|_{K_{a}}}\right)(a)$. Then we select a sequence of elements $\left\{K_{1}, \cdots, K_{J_{a}}\right\} \subset \mathcal{T}_{h}(a)$ such that $K_{1}=K, K_{J_{a}}=K_{a}$, and $f_{j}=K_{j} \cap K_{j+1}$ is a common $(d-1)$-dimensional simplex of $K_{j}$ and $K_{j+1}$. We write the right-hand side of the above inequality as the telescopic sum:

$$
|\nabla \phi(a)|^{2} \leq \sum_{j=1}^{J_{a}-1}\left|\nabla w_{\left.\right|_{K_{j}}}(a)-\nabla w_{\left.\right|_{K_{j+1}}}(a)\right|^{2} .
$$

Note that $w$ is continuous across $f_{j}$, we write

$$
|\nabla \phi(a)|^{2} \leq C \sum_{f \in \mathcal{F}_{a}^{I}} h_{F}^{-2}\left\|\llbracket \partial_{n_{f}} w \rrbracket\right\|_{L^{2}(f)}^{2} .
$$


If the node $a \in \mathcal{V}_{h}^{\#}$, the edges $\mathcal{E}_{a} \subset \mathcal{E}_{h}^{B}(a)$ provide a basis of $\mathbb{R}^{d}$, we have

$$
|\nabla \phi(a)| \leq C \sum_{e \in \mathcal{E}_{a}}\left|\partial_{e} w_{\left.\right|_{K}}(a)-\partial_{e} w_{\left.\right|_{K_{e}}}(a)\right|,
$$

where $\partial_{e} w=e \cdot \nabla w, K_{e}$ is a simplex with the boundary edge $e$, and we employ the fact $w_{l_{e}}=0$. Proceeding along the same line by connecting $K$ to $K_{e}$ with a sequence of simplices, we obtain

$$
|\nabla \phi(a)|^{2} \leq C \sum_{f \in \mathcal{F}_{a}^{I}}\left\|\llbracket \partial w / \partial_{n_{f}} \rrbracket\right\|_{L^{2}(f)}^{2} .
$$

Substituting (3.8), (3.9) into (3.7), we obtain

$$
\|\phi\|_{L^{2}(K)} \leq C h_{K}^{3 / 2} \sum_{a \in \mathcal{V}_{K}} \sum_{f \in \mathcal{F}_{a}^{I}}\left\|\llbracket \partial_{n} w \rrbracket\right\|_{L^{2}(f)},
$$

where we have used the fact that $h_{K} \simeq h_{f}$ because $\mathcal{T}_{h}$ is locally quasi-uniform.

Therefore, using the above inequality, the estimate for the Scott-Zhang interpolant, the inverse inequality and the trace inequality (2.25), we obtain

$$
\begin{aligned}
\left\|v-I_{h} v\right\|_{H^{k}(K)} & \leq\left\|v-\Pi_{C} v\right\|_{H^{k}(K)}+\left\|\Pi_{C} v-\Pi_{h} \Pi_{C} v\right\|_{H^{k}(K)} \\
& \leq C h_{K}^{m-k}\|v\|_{H^{m}(K)}+C h_{K}^{\frac{3}{2}-k} \sum_{a \in \mathcal{V}_{K}} \sum_{f \in \mathcal{F}_{a}^{I}}\left\|\partial_{n}\left(\Pi_{C} v-v\right)\right\|_{L^{2}(f)} \\
& \leq C h_{K}^{m-k}\|v\|_{H^{m}\left(\mathcal{T}_{K}\right)},
\end{aligned}
$$

where $\mathcal{T}_{K}=\cup_{a \in \mathcal{V}_{K}} \mathcal{T}_{h}(a)$ is the local element star of $K$. Summing up the above inequalities for $K \in \mathcal{T}_{h}$, we obtain (3.5).

Next, for any $v \in H_{0}^{2}(\Omega) \cap H^{m}(\Omega)$, the estimate (3.6) may be proceeded along the same line that leads to (3.5), we omit the details.

\section{Error Estimate for Less Smooth Solution}

The standard error estimate argument is valid under the regularity assumption $u \in H^{s}(\Omega)$ with $s \geq 3$, which is usually invalid for the point loading or nonconvex domain [7. In this part we shall exploit enriching operator to derive a new error estimate for Problem (1.1) with less smooth solution. The enriching operator measures the distance between $V_{h}$ and $H^{2}(\Omega)$, which was firstly introduced by Brenner 9, 10, to analyze nonconforming elements in the context of fast solvers. Li, Ming AND Shi 28, Lemma 4.1] have constructed an enriching operator for the quadratic Specht triangle and have obtained optimal error estimate for approximating the biharmonc problems with rough solution. The construction and the proof therein equally applies to the Specht triangle. We also note that there are many different type enriching operators for Morley's triangle; See, e.g., 22, 43. In what follows, we shall construct such enriching operator for the NZT tetrahedron with the aid of the ninth polynomial $C^{1}$-conforming element introduced by ZHANG 47. The enriching operator $E_{h}: X_{h}^{0} \rightarrow H_{0}^{2}(\Omega)$ is defined as follows. 
(1) For any $a \in \mathcal{V}_{h}^{I}$, we fix a element $K_{a}$ from element star $\mathcal{T}_{h}(a)$,

$$
\left(\nabla^{\alpha} E_{h} v\right)(a):=\left(\left.\nabla^{\alpha} v\right|_{K_{a}}\right)(a), K_{a} \in \mathcal{T}_{h}(a),|\alpha| \leq 4
$$

(2) For any edge $e \in \mathcal{E}_{h}^{I}$, we equip the edge $e$ with unit direction vectors $\mathcal{S}_{e}=\left\{s_{1}, s_{2}\right\}$, where $s_{i}$ is orthogonal to $e$, and $\mathcal{S}_{e} \cup\{e\}$ provides a basis of $\mathbb{R}^{3}$. We fix an element from element star $K_{e} \in \mathcal{T}_{h}(e)$. For $a$ the middle point of an edge $e$,

$$
\partial_{s_{i}} E_{h} v(a):=\left(\left.\partial_{s_{i}} v\right|_{K_{e}}\right)(a), \quad s_{i} \in \mathcal{S}_{e}
$$

For $b$ and $c$ the equally distributed interior points of the edge $e$, for $p=b, c$,

$$
\frac{\partial^{2} E_{h} v}{\partial s_{i} \partial s_{j}}(p)=\frac{\left.\partial^{2} v\right|_{K_{e}}}{\partial s_{i} \partial s_{j}}(p), \quad s_{i}, s_{j} \in \mathcal{S}_{e} .
$$

(3) For any $f \in \mathcal{F}_{h}^{I}$, and for any $w \mathbb{P}_{0}(f)$, we define

$$
\int_{f} E_{h} v w \mathrm{~d} \sigma(x):=\int_{f} v w \mathrm{~d} \sigma(x) .
$$

and for any $w \in \mathbb{P}_{2}(f)$,

$$
\int_{f} \partial_{n}\left(E_{h} v\right) w \mathrm{~d} \sigma(x):=\int_{f}\left\{\left\{\partial_{n} v\right\}\right\} w \mathrm{~d} \sigma(x) .
$$

(4) For any $K \in \mathcal{T}_{h}$, and for $w \in \mathbb{P}_{1}(K)$,

$$
\int_{K} E_{h} v w \mathrm{~d} x:=\int_{K} v w \mathrm{~d} x .
$$

(5) All the degrees of freedom of $E_{h} v$ vanish on the $\partial \Omega$.

We summarize the properties of the enriching operator in the following lemma.

Lemma 4.1. The enriching operator $E_{h}$ has the following properties:

(1) Petrov-Galerkin orthogonality: For any $v \in V_{h}$,

$$
a_{h}\left(v-E_{h} v, w\right)=0 \quad \text { for all } \quad w \in W_{h},
$$

where $W_{h}$ is the tensorized $\mathbb{P}_{2}$ element space with vanishing trace.

(2) $E_{h}$ is stable in the sense that

$$
\left\|E_{h} v\right\|_{\iota, h} \leq \alpha\|v\|_{\iota, h} \quad \text { for all } \quad v \in V_{h} .
$$

(3) For any $v \in V_{h}$, we have

$$
\left\|\nabla_{h}^{k}\left(v-E_{h} v\right)\right\|_{L^{2}} \leq \beta h^{j-k}\left\|\nabla_{h}^{j} v\right\|_{L^{2}}, \quad 0 \leq k \leq j \leq 2 .
$$

The stability estimate (4.5) is a direct consequence of (4.6). We only give the details for (4.4) and (4.6). 
Proof. For any $v \in V_{h}$ and $w \in W_{h}$, integration by parts, we obtain

$$
\begin{aligned}
a_{h}(v & \left.-E_{h} v, w\right)=\sum_{K \in \mathcal{T}_{h}} \int_{\partial K} \partial_{n}\left(v-E_{h} v\right) M_{n n}(w) \mathrm{d} \sigma(x) \\
& =\sum_{F \in \mathcal{F}_{h}^{I}} \int_{F}\left(\llbracket \partial_{n}\left(v-E_{h} v\right) \rrbracket\left\{\left\{M_{n n}(w)\right\}\right\}+\left\{\left\{\partial_{n}\left(v-E_{h} v\right)\right\}\right\} \llbracket M_{n n}(w) \rrbracket\right) \mathrm{d} \sigma(x) .
\end{aligned}
$$

Using the fact that $M_{n n}(w)=n^{\mathrm{T}} \cdot(\mathbb{D} \nabla \epsilon(w)) \cdot n \in \mathbb{P}_{0}(K)$,

$$
\llbracket \partial_{n}\left(v-E_{h} v\right) \rrbracket=\llbracket \partial_{n} v \rrbracket \quad \text { and } \quad\left\{\left\{\partial_{n}\left(v-E_{h} v\right)\right\}\right\}=\left\{\left\{\partial_{n} v\right\}\right\}-\partial_{n} E_{h} v,
$$

and (4.3), we obtain (4.4).

As to (4.6), we only prove $k=0$, and the general case may be resorting to the inverse inequality because $v$ and $E_{h} v$ are piecewise polynomials.

For any element $K \in \mathcal{T}_{h}$, we let $\mathcal{N}, \mathcal{E}, \mathcal{F}$ and $\mathcal{V}$ be the set of the nodal variables, edge variables, face variable, and the set of the volume variables of $\mathbb{P}_{9}$ conforming element, respectively. For any $v \in V_{h}, v-E_{h} v \in \mathbb{P}_{9}$, and it follows from the scaling argument that

$$
\begin{aligned}
\left\|v-E_{h} v\right\|_{L^{2}(K)}^{2} \leq & C \sum_{N \in \mathcal{N}(K)} h_{K}^{3+2 \operatorname{order}(N)}\left(N\left(v-E_{h} v\right)\right)^{2} \\
& +C \sum_{E \in \mathcal{E}(K)} h_{K}^{3+2 \operatorname{order}(E)}\left(E\left(v-E_{h} v\right)\right)^{2} \\
& +C \sum_{F \in \mathcal{F}(K)} h_{K}^{3+2 \operatorname{order}(F)}\left(F\left(v-E_{h} v\right)\right)^{2} \\
& +C \sum_{V \in \mathcal{V}(K)} h_{K}^{3+2 \operatorname{order}(V)}\left(V\left(v-E_{h} v\right)\right)^{2} \\
= & : I_{1}+\cdots+I_{4},
\end{aligned}
$$

where $\operatorname{order}(N)$ is the order of the differentiation in the definition of $N$, and the same rule applies to $\operatorname{order}(E), \operatorname{order}(F)$ and $\operatorname{order}(V)$. It is clear that $I_{4}=0$ because $V(v)=V\left(E_{h} v\right)$.

Note that we have $N\left(v-E_{h} v\right)=0$ when $\operatorname{order}(N)=0,1$. It remains to estimate the case $\operatorname{order}(N)=2,3,4$. By the standard inverse estimate, we obtain

$$
\begin{aligned}
I_{1} & \leq C \sum_{l=2}^{4} \sum_{p \in \mathcal{V}_{K}} h_{K^{\prime}}^{3+2 l} \sum_{K^{\prime} \in \mathcal{T}_{h}(p)}\left\|\nabla^{l} v\right\|_{L^{\infty}\left(K^{\prime}\right)}^{2} \\
& \leq C \sum_{l=2}^{4} \sum_{p \in \mathcal{V}_{K}} h_{K^{\prime}}^{3+2 l} \sum_{K^{\prime} \in \mathcal{T}_{h}(p)} h_{K^{\prime}}^{1-2 l}\left\|\nabla^{2} v\right\|_{L^{2}\left(K^{\prime}\right)}^{2} \\
& \leq C \sum_{K^{\prime} \in \mathcal{T}_{K}} h_{K^{\prime}}^{4}\left\|\nabla_{h}^{2} v\right\|_{L^{2}\left(K^{\prime}\right)}^{2},
\end{aligned}
$$

where $\mathcal{V}_{K}$ is the set containing all the vertices of $K$, and $\mathcal{T}_{K}=\cup_{p \in \mathcal{V}_{K}} \mathcal{T}_{h}(p)$ is local element star of $K$. 
Next, we denote $I_{2}^{1}$ for the term in $I_{2}$ with $\operatorname{order}(E)=1$, and $I_{2}^{2}$ for the term in $I_{2}$ with $\operatorname{order}(E)=2$. For $\operatorname{order}(E)=1$ and any edge $e \in \mathcal{E}_{h}$, by the (4.1), we have

$$
\left|E\left(v-E_{h} v\right)\right|^{2} \leq\left|\left(\left.\nabla v\right|_{K}\right)(a)-\left(\left.\nabla v\right|_{K_{e}}\right)(a)\right|^{2} .
$$

We select a sequence of elements $\left\{K_{1}, \cdots, K_{J_{e}}\right\} \subset \mathcal{T}_{h}(e)$ such that $K_{1}=K, K_{J_{e}}=$ $K_{e}$, and $f_{j}=K_{j} \cap K_{j+1}$ is a common face of $K_{j}$ and $K_{j+1}$. We write the right-hand side of the above inequality as the telescopic sum:

$$
\begin{aligned}
\left|\left(\left.\nabla v\right|_{K}\right)(a)-\left(\left.\nabla v\right|_{K_{e}}\right)(a)\right|^{2} & =\sum_{j=1}^{J_{e}-1}\left|\partial_{n} v\right|_{K_{j}}(a)-\left.\left.\partial_{n} v\right|_{K_{j+1}}(a)\right|^{2} \\
& \leq C \sum_{f \in \mathcal{F}_{h}(e)}|f|^{-1}\left\|\llbracket \partial_{n} v \rrbracket\right\|_{L^{2}(f)}^{2} .
\end{aligned}
$$

Note that $\int_{f} \llbracket \partial_{n} v \rrbracket \mathrm{d} \sigma(x)=0$, we use the Poincaré inequality and the trace inequality (2.26) to obtain

$$
\begin{aligned}
I_{2}^{1} & \leq C \sum_{e \in \mathcal{E}_{K}} \sum_{f \in \mathcal{F}_{h}(e)} h_{K}^{5}|f|^{-1}\left\|\llbracket \partial_{n} v \rrbracket\right\|_{L^{2}(f)}^{2} \\
& \leq C \sum_{e \in \mathcal{E}_{K}} \sum_{f \in \mathcal{F}_{h}(e)} h_{K}^{5}\left\|\nabla \llbracket \partial_{n} v \rrbracket\right\|_{L^{2}(f)}^{2} \\
& \leq C \sum_{K^{\prime} \in \mathcal{T}_{K}} h_{K}^{4}\left\|\nabla^{2} v\right\|_{L^{2}\left(K^{\prime}\right)}^{2},
\end{aligned}
$$

where $\mathcal{E}_{K}$ is the set containing all edges of $K$.

For $b$ and $c$ the equally distributed interior points of an edge $e$, and $p=b, c$, by (4.2) and the inverse estimate, we obtain

$$
I_{2}^{2} \leq C \sum_{e \in \mathcal{E}_{K}} \sum_{K^{\prime} \in \mathcal{T}_{h}(e)} h_{K^{\prime}}^{7}\left\|\nabla^{2} v\right\|_{L^{\infty}\left(K^{\prime}\right)}^{2} \leq C \sum_{K^{\prime} \in \mathcal{T}_{K}} h_{K^{\prime}}^{4}\left\|\nabla^{2} v\right\|_{L^{2}\left(K^{\prime}\right)}^{2}
$$

Last, we have $F\left(v-E_{h} v\right)=0$ when $\operatorname{order}(F)=0$. By (4.3), and a standard scaling argument gives

$$
I_{3} \leq C \sum_{f \in \mathcal{F}_{K}} h_{F}^{5}\left|f_{f} \llbracket \partial_{n} w \rrbracket \mathrm{d} \sigma(x)\right|^{2} \leq C \sum_{f \in \mathcal{F}_{K}} h_{f}^{5}|f|^{-1}\left\|\llbracket \partial_{n} w \rrbracket\right\|_{L^{2}(f)}^{2},
$$

where $\mathcal{F}_{K}$ is the set containing all face of $K$. Note that $\int_{f} \llbracket \partial_{n} v \rrbracket \mathrm{d} \sigma(x)=0$ for any face $f \in \mathcal{F}_{h}$, using Poincaré inequality and the trace inequality again, we obtain

$$
I_{3} \leq C \sum_{K^{\prime} \in \mathcal{T}_{K}} h_{K^{\prime}}^{4}\left\|\nabla^{2} v\right\|_{L^{2}\left(K^{\prime}\right)}^{2} .
$$

Summing up the estimates for $I_{1}, \cdots, I_{4}$, we obtain

$$
\left\|v-E_{h} v\right\|_{L^{2}(K)}^{2} \leq C \sum_{K^{\prime} \in \mathcal{T}_{K}} h_{K^{\prime}}^{4}\left\|\nabla^{2} v\right\|_{L^{2}\left(K^{\prime}\right)}^{2} \leq C \sum_{K^{\prime} \in \mathcal{T}_{K}} h_{K^{\prime}}^{2 j}\left\|\nabla^{j} v\right\|_{L^{2}\left(K^{\prime}\right)}^{2} .
$$

Summing up all the elements for $K \in \mathcal{T}_{h}$, we obtain [4.6). 
Theorem 4.2. Let $u$ and $u_{h}$ be the solutions of Problem (2.2) and Problem (3.2), respectively. Then

$$
\left\|u-u_{h}\right\|_{\iota, h} \leq(1+\alpha)\left(2 \inf _{v \in V_{h}}\|u-v\|_{\iota, h}+\inf _{w \in W_{h}}\|u-w\|_{\iota, h}\right)+\beta \operatorname{Osc}(f),
$$

where the oscillation of $f$ is defined as

$$
\operatorname{Osc}(f):=\left(\sum_{K \in \mathcal{T}_{h}} h_{K}^{2} \inf _{\bar{f} \in \mathbb{P}_{1}(K)}\|f-\bar{f}\|_{L^{2}(K)}^{2}\right)^{1 / 2} .
$$

Proof. For any $v \in V_{h}$, we denote $w=v-u_{h}$ and $E_{h} w=\left(E_{h} w_{1}, \cdots, E_{h} w_{d}\right)$. By the Galerkin orthogonality of the enriching operator (4.4), we obtain, for any $z \in W_{h}$,

$$
\begin{aligned}
\|w\|_{\iota, h}^{2} & =a_{h}(v, w)-a_{h}\left(u_{h}, w\right)=a_{h}\left(v, w-E_{h} w\right)+a_{h}\left(v, E_{h} w\right)-(f, w) \\
& =a_{h}\left(v-z, w-E_{h} w\right)+a_{h}\left(v-u, E_{h} w\right)+\left(f, E_{h} w-w\right) \\
& =a_{h}\left(v-z, w-E_{h} w\right)+a_{h}\left(v-u, E_{h} w\right)+\left(f-\bar{f}, E_{h} w-w\right),
\end{aligned}
$$

where we have used (4.3) 3 in the last step. The energy estimate (4.7) follows from (4.5) and (4.6) with $k=0, j=1$ and the triangle inequality and the estimate

$$
\left|a_{h}\left(v-z, w-E_{h} w\right)\right| \leq(1+\alpha)\left(\|u-v\|_{\iota, h}+\|u-z\|_{\iota, h}\right)\|w\|_{\iota, h} .
$$

We are ready to derive the rate of convergence for the Specht triangle and the NZT tetrahedron.

Theorem 4.3. Let $u$ and $u_{h}$ be the solutions of problem (2.2) and problem (3.2), if the Hypothesis 2.3 is true, then

$$
\left\|u-u_{h}\right\|_{\iota, h} \leq C h^{1 / 2}\|f\|_{L^{2}} .
$$

If $u \in H^{3}(\Omega)$, then

$$
\left\|u-u_{h}\right\|_{\iota, h} \leq C\left(h^{2}+\iota^{2} h\right)\|u\|_{H^{3}} .
$$

Proof. By (3.6), and combining the regularity estimates (2.16) and (2.17), we have

$$
\inf _{v \in V_{h}}\|u-v\|_{\iota, h} \leq \iota\left\|\nabla_{h}^{2}\left(u-I_{h}^{0} u\right)\right\|_{L^{2}}+\left\|\nabla\left(u-I_{h}^{0} u\right)\right\|_{L^{2}} \leq C h^{1 / 2}\|f\|_{L^{2}} .
$$

By the interpolation error estimate of SCOTT-ZHANG interpolant [38, we obtain

$$
\inf _{v \in W_{h}}\|u-v\|_{\iota, h} \leq \iota\left\|\nabla_{h}^{2}\left(u-\Pi_{C} u\right)\right\|_{L^{2}}+\left\|\nabla\left(u-\Pi_{C} u\right)\right\|_{L^{2}} \leq C h^{1 / 2}\|f\|_{L^{2}} .
$$

It is clear that

$$
\operatorname{Osc}(f) \leq C h\|f\|_{L^{2}} .
$$

Combining all the above inequalities and (4.7), we obtain (4.9).

The estimate (4.10) may be proved in a standard manner, we omit the details. 


\section{NUMERICAL EXPERIMENTS}

In this part, we test the accuracy of the Specht triangle and the NZT tetrahedorn and the numerical pollution effect for a solution with strong boundary layer. In all the examples, we let $\Omega=(0,1)^{d}$ and set $\lambda=10, \mu=1$. For $d=2$, the initial unstructured mesh consists of 220 triangles and 127 vertices, and the maximum mesh size is $h=1 / 8$; See Figure $1_{\imath}$. For $d=3$, we construct initial mesh by splitting origin cube into 512 small cubes, and each small cube is divided into 6 tetrahedrons; See Figure 1,

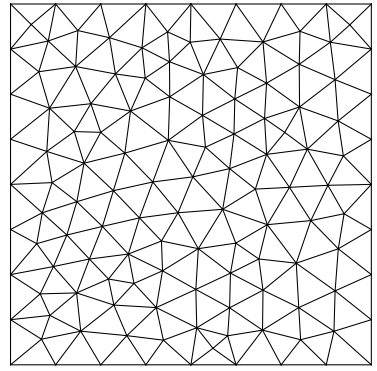

(a)

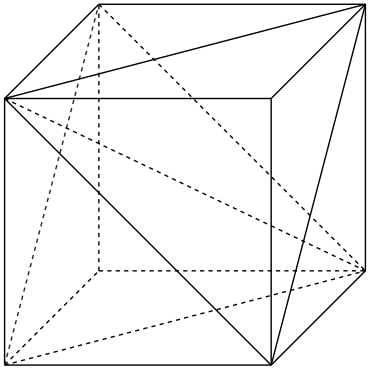

(b)

Figure 1. Plots of meshes: (a) $d=2$; (b) $d=3$.

Throughout the simulation, we employ the analytic basis functions for the Specht triangle [41, 48, and the NZT tetrahedron 45. The computation for the NZT tetrahedron is performed in a parallel hierarchical grid platform (PHG) 46]

5.1. Example for smooth solution. This example is to test the accuracy of the elements. We let $u=\left(u_{1}, u_{2}, u_{3}\right)$ with

$$
\begin{aligned}
& u_{1}=\prod_{i=1}^{d}\left(\exp \left(\cos 2 \pi x_{i}\right)-\exp (1)\right), u_{2}=\prod_{i=1}^{d}\left(\cos 2 \pi x_{i}-1\right), \\
& u_{3}=\prod_{i=1}^{d} x_{i}^{2}\left(x_{i}-1\right)^{2} .
\end{aligned}
$$

The source term $f$ is computed by $(1.1)_{1}$. For $d=2$, we drop the third component $u_{3}$. This solution is smooth, and we measure the rates of convergence in the relative energy norm $\left\|u-u_{h}\right\|_{\iota, h} /\|u\|_{\iota, h}$ for different $\iota$. We report the rates of convergence for the Specht triangle and the NZT tetrahedorn in Table 1 and Table 2 respectively. We observe that the rates of convergence appear to be linear when $\iota$ is large, while it turns out to be quadratic when $\iota$ is close to zero, which is consistent with the theoretical predications (4.10).

\footnotetext{
${ }^{1}$ http://lsec.cc.ac.cn/phg
} 
NONCONFORMING ELEMENTS FOR STRAIN GRADIENT MODEL

TABLE 1. Rate of convergence of the Specht triangle.

\begin{tabular}{lllllll}
\hline$\iota h$ & $1 / 8$ & $1 / 16$ & $1 / 32$ & $1 / 64$ & $1 / 128$ & $1 / 256$ \\
\hline $1 \mathrm{e}+0$ & $1.99 \mathrm{e}-01$ & $9.87 \mathrm{e}-02$ & $4.80 \mathrm{e}-02$ & $2.36 \mathrm{e}-02$ & $1.17 \mathrm{e}-02$ & $5.85 \mathrm{e}-03$ \\
rate & & 1.01 & 1.04 & 1.02 & 1.01 & 1.00 \\
$1 \mathrm{e}-2$ & $3.16 \mathrm{e}-02$ & $1.21 \mathrm{e}-02$ & $5.30 \mathrm{e}-03$ & $2.53 \mathrm{e}-03$ & $1.25 \mathrm{e}-03$ & $6.21 \mathrm{e}-04$ \\
rate & & 1.39 & 1.19 & 1.07 & 1.02 & 1.01 \\
$1 \mathrm{e}-4$ & $2.20 \mathrm{e}-02$ & $5.57 \mathrm{e}-03$ & $1.39 \mathrm{e}-03$ & $3.48 \mathrm{e}-04$ & $8.75 \mathrm{e}-05$ & $2.26 \mathrm{e}-05$ \\
rate & & 1.98 & 2.00 & 2.00 & 1.99 & 1.95 \\
$1 \mathrm{e}-6$ & $2.20 \mathrm{e}-02$ & $5.57 \mathrm{e}-03$ & $1.39 \mathrm{e}-03$ & $3.47 \mathrm{e}-04$ & $8.65 \mathrm{e}-05$ & $2.16 \mathrm{e}-05$ \\
rate & & 1.98 & 2.00 & 2.00 & 2.00 & 2.00 \\
\hline
\end{tabular}

TABLE 2. Rates of convergence of the NZT tetrahedorn.

\begin{tabular}{llllll}
\hline$\iota \backslash h$ & $1 / 8$ & $1 / 16$ & $1 / 32$ & $1 / 64$ & $1 / 128$ \\
\hline $1 \mathrm{e}+0$ & $1.02 \mathrm{e}-01$ & $7.54 \mathrm{e}-01$ & $5.05 \mathrm{e}-01$ & $2.84 \mathrm{e}-01$ & $1.47 \mathrm{e}-01$ \\
rate & & 0.43 & 0.58 & 0.83 & 0.95 \\
$1 \mathrm{e}-2$ & $5.12 \mathrm{e}-01$ & $2.28 \mathrm{e}-01$ & $8.99 \mathrm{e}-02$ & $3.57 \mathrm{e}-02$ & $1.55 \mathrm{e}-02$ \\
rate & & 1.17 & 1.34 & 1.33 & 1.20 \\
$1 \mathrm{e}-4$ & $3.03 \mathrm{e}-01$ & $7.14 \mathrm{e}-02$ & $1.79 \mathrm{e}-02$ & $4.66 \mathrm{e}-03$ & $1.27 \mathrm{e}-03$ \\
rate & & 2.08 & 1.99 & 1.94 & 1.89 \\
$1 \mathrm{e}-6$ & $3.01 \mathrm{e}-01$ & $6.98 \mathrm{e}-02$ & $1.71 \mathrm{e}-02$ & $4.26 \mathrm{e}-03$ & $1.07 \mathrm{e}-03$ \\
rate & & 2.11 & 2.03 & 2.00 & 1.99 \\
\hline
\end{tabular}

5.2. Example with boundary layer. In this example, we test the performance of both elements to resolve a solution with strong boundary layer, such boundary layer is one of the difficulty for the strain gradient elasticity model, and we refer to [17] for a one-dimensional example with analytical expression, the following construction is based on that example. We construct a displacement field $u=\left(u_{1}, u_{2}, u_{3}\right)$ with a layer as

$$
\begin{aligned}
& u_{1}=\prod_{i=1}^{d}\left(\exp \left(\sin \pi x_{i}\right)-1-\varphi\left(x_{i}\right)\right), \quad u_{2}=\prod_{i=1}^{d}\left(\sin \pi x_{i}-\varphi\left(x_{i}\right)\right), \\
& u_{3}=\prod_{i=1}^{d}\left(-\pi x_{i}\left(x_{i}-1\right)-\varphi\left(x_{i}\right)\right)
\end{aligned}
$$

with

$$
\varphi(x)=\pi \iota \frac{\cosh [1 / 2 \iota]-\cosh [(2 x-1) / 2 \iota]}{\sinh [1 / 2 \iota]} .
$$


A direct calculation gives

$$
\lim _{\iota \rightarrow 0} u=u_{0}=\left(\prod_{i=1}^{d} \exp \left(\sin \pi x_{i}\right)-1, \prod_{i=1}^{d} \sin \pi x_{i}, \prod_{i=1}^{d} \pi x_{i}\left(1-x_{i}\right)\right),
$$

with $\left.u_{0}\right|_{\partial \Omega}=0$ and $\left.\partial_{n} u_{0}\right|_{\partial \Omega} \neq 0$. It is clear that $\partial_{n} u$ has boundary layers. The source term $f$ is also computed from (1.1) $)_{1}$. We report the rates of convergence for the elements in the relative energy norm $\left\|u-u_{h}\right\|_{\iota, h} /\|u\|_{\iota, h}$ with $\iota=10^{-6}$ in Table 3. The half order rates of convergence are observed for both elements, which are consistent with the theoretical predictions (4.10).

TABLE 3. Rates of convergence for $\iota=10^{-6}$.

\begin{tabular}{lllllll}
\hline$h$ & $1 / 8$ & $1 / 16$ & $1 / 32$ & $1 / 64$ & $1 / 128$ & $1 / 256$ \\
\hline NZT & $2.74 \mathrm{e}-01$ & $1.73 \mathrm{e}-02$ & $1.18 \mathrm{e}-01$ & $8.24 \mathrm{e}-02$ & $5.80 \mathrm{e}-02$ & $4.10 \mathrm{e}-2$ \\
rate & & 0.66 & 0.56 & 0.52 & 0.51 & 0.50 \\
Specht & $1.57 \mathrm{e}-01$ & $1.10 \mathrm{e}-01$ & $7.70 \mathrm{e}-02$ & $5.42 \mathrm{e}-02$ & $3.82 \mathrm{e}-02$ & $2.70 \mathrm{e}-02$ \\
rate & & 0.51 & 0.51 & 0.51 & 0.50 & 0.50 \\
\hline
\end{tabular}

\section{Conclusion}

We prove a new $\mathrm{H}^{2}$-Korn's inequality and a new broken $\mathrm{H}^{2}$-Korn's inequality. The former is crucial for the well-posedness of a strain gradient elasticity model, while the latter motivates us to construct robust nonconforming elements for this model, and the elements are simpler than the known elements in the literature; See, e.g., 27. With the aid of the new regularized interpolant and the enriching operator, we proved that the tensor product of the Specht triangle and the NZT tetrahedron converges uniformly with respect to the small materials parameter under the minimal smoothness assumption on the solution. Moreover, the technicalities may also be used to derive shaper error bounds for the elements in [35, 42, 24]. Guided by the broken $\mathrm{H}^{2}$-Korn's inequality, we can design robust elements for the nonlinear strain gradient elastic models, thin beam and thin plate with strain gradient effect in [20, 17] by combining the tricks in [8, 33] and the machinery developed in this article, which will be left for further pursuit.

\section{REFERENCES}

1. H. Abel, M. Giovanna Mora, and S. Müller, Large time existence for thin vibrating plates, Comm. PDEs 36 (2011), 2062-2102.

2. R.A. Adams and J.J.F. Fournier, Sobolev Spaces, Academic Press, 2nd ed., 2003.

3. S. Agmon, L. Douglis, and A. Nirenberg, Estimates near the boundary for solutions of elliptic partial differential equations satisfying general boundary conditions II, Comm. Pure Appl. Math. 17 (1964), 35-92. 
4. S.B. Altan and E.C. Aifantis, On the structure of the mode III crack-tip in gradient elasticity, Scripta Metal. Mater. 26 (1992), 319-324.

5. J.H. Argyris, I. Fried, and D.W. Scharpf, The Tuba family of plate elements for the matrix displacement method, The Aero. J. Roy. Aero. Soc. 72 (1968), 701-709.

6. H. Askes and E.C. Aifantis, Gradient elasticity in statics and dynamics: An overview of formulations, length scale identification procedures, finite element implementations and new results, Inter. J. Solids and Struc. 48 (2011), 1962-1990.

7. H. Blum and R. Rannacher, On the boundary value problem of the biharmonic operator on domains with angular corners, Math. Methods Appl. Sci. 2 (1980), 556-581.

8. D. Braess and P.B. Ming, A finite element method for nearly incompressible elasticity problem, Math. Comp. 74 (2005), 25-52.

9. S.-C. Brenner, Two level additive Schwartz preconditioners for nonconforming finite elements, In Domain Decomposition Methods in Scientific and Engineering Computing, Edited by D.E. Keys and J. Xu., Amer. Math. Soc., Providence, Comtemporary Mathematics, Vol. 180, 1994, pp. 9-14.

10. - Two level additive Schwartz preconditioners for nonconforming finite elements, Math. Comp. 65 (1996), 897-921.

11. S.C. Brenner, Korn's inequalities for piecewise $H^{1}$ vector fields, Math. Comp. 73 (2004), 1067-1087.

12. S.C. Brenner and M. Neilan, A $C^{0}$ interior penalty method for a fourth order elliptic singular perturbation problem, SIAM J. Numer. Anal. 49 (2011), 869-892.

13. S.C. Brenner and L.R. Scott, The Mathematical Theory of Finite Element Methods, Springer Science + Buiness Media LLC. 3rd eds., 2008.

14. P.G. Ciarlet, The Finite Element Method for Elliptic Problems, North-Holland, Amsterdam, 1978.

15. L Linear and Nonlinear Functional Analysis with Applications, SIAM, 2013.

16. P.G. Ciarlet and P.-A. Raviart, General Lagrange and Hermite interpolation in $R^{n}$ with applications to finite element methods, Arch. Rational Mech. Anal. 46 (1972), 177-199.

17. G. Engel, K. Garikipati, T.J.R. Hughes, M.G. Larsson, L. Mazzei, and R.L. Taylor, Continuous/discontinuous finite element approximations of fourth-order elliptic problems in structural and continuum mechanics with applications to thin beams and plates, and strain gradient elasticity, Comput. Methods Appl. Mech. Engrg. 191 (2002), 3669-3750.

18. A.C. Eringen, Nonlinear Continuum Field Theories, Springer-Verlag, New York, 2002.

19. G.E. Exadaktylos and E.C. Aifantis, Two and three dimensional crack problems in gradient elasticity, J. Mech. Behav. Mater. 7 (1996), 93-118.

20. P. Fisher, J. Mergheim, and P. Steinmann, On the $C^{1}$ continuous discretization of non-linear gradient elasticity: a comparison of NEM and FEM based on Bernstein-Bézier patches, Int. J. Numer. Meth. Engrg. 82 (2010), 1282-1307.

21. N.A. Fleck and J.W. Hutchinson, Strain gradient plasticity, Advances in Applied Mechanics, Vol. 33, Academic Press, 1997, pp. 295-361.

22. D. Gallistl, Morley finite element method for the eigenvalue of the biharmonic operator, IMA J. Numer. Anal. 35 (2015), 1779-1811.

23. P. Grisvard, Elliptic Problems in Nonsmooth Domains, Pitman, Boston, 1985.

24. J. Guzmán, D. Leykekhman, and M. Neilan, A family of non-conforming elements and the analysis of Nitsche's method for a singularly perturbed fourth order problem, Calcolo 49 (2012), 95-125. 
25. A. Korn, Solution générale du problème d'équilibre dans la théorie de l'élasticité dans le cas où les efforts sont donnés à la surface, Ann. Fac. Sci. Toulouse Sci. Math. Sci. Phys. (2) 10 (1908), 165-269.

26. — Über einige ungleichungen, welche in der Theorie der elastischen und elektrischen Schwingungen eine Rolle spielen, Bull. Intern. Cracov. Akad. Umiejetnosci (Classe Sci. Math. Nat.) (1909), 706-724.

27. H.L. Li, P.B. Ming, and Z.-C. Shi, Two robust nonconforming $H^{2}$-elements for linear strain gradient elasticity, Numer. Math. 137 (2017), 691-711.

28.

29. Y.L. Liao and P.B. Ming, A family of nonconforming rectangular elements for strain gradient elasticity, Adv. Appl. Math. Mech. 11 (2019), 1263-1286.

30. K.A. Mardal and R. Winther, An observation on Korn's inequality for nonconforming finite element methods, Math. Comp. 75 (2006), 1-6.

31. V. Maźya and J. Rossmann, Elliptic Equations in Polyhedral Domains, American Mathematical Society, Providence, RI, 2010.

32. R.D. Mindlin, Microstructure in linear elasticity, Arch. Rational Mech. Anal 10 (1964), 51-78.

33. P.B. Ming and Z.-C. Shi, Analysis of some low order quadrilateral Reissner-Mindlin plate elements, Math. Comp. 75 (2006), 1043-1065.

34. M. Neilan and M.H. Wu, Discrete Miranda-Talenti estimates and applications to linear and nonlinear PDEs, J. Comput. Appl. Math. 356 (2019), 358-376.

35. T.K. Nilssen, X.C. Tai, and R. Winther, A robust nonconforming $H^{2}$-element, Math. Comp. 70 (2001), 489-505.

36. S.-A. Papanastasiou, A. Zervos, and I. Vardoulakis, A three-dimensional $C^{1}$ finite element for gradient elasticity, Int. J. Numer. Meth. Engrg. 135 (2009), 1396-1415.

37. C.Q. Ru and E.C. Aifantis, A simple approach to solve boundary-value problems in gradient elasticity, Acta Mech. 101 (1993), 59-68.

38. L.R. Scott and S.Y. Zhang, Finite element interpolation of nonsmooth functions satisfying boundary conditions, Math. Comp. 54 (1990), 483-493.

39. B. Semper, Conforming finite element approximations for a fourth-order singular perturbation problem, SIAM J. Numer. Anal. 29 (1992), 1043-1058.

40. __ Locking in finite-element approximations to long thin extensible beams, IMA J. Numer. Anal. 14 (1994), 97-109.

41. B. Specht, Modified shape functions for the three node plate bending element passing the patch test, Int. J. Numer. Meth. Engrg. 28 (1988), 705-715.

42. X.C. Tai and R. Winther, A discrete de Rham complex with enhanced smoothness, Calcolo 43 (2006), 287-306.

43. A. Veeser and P. Zanotti, Quasi-optimal nonconforming methods for symmetric elliptic problems. II-overconsistency and classical nonconforming elements, SIAM J. Numer. Anal. 57 (2019), 266-292.

44. H.Y. Wang, Nonconforming tetrahedron elements for strain gradient elasticity model, 2020, $\mathrm{Ph}$. D. thesis, Chinese Academy of Sciences.

45. M. Wang, Z.-C. Shi, and J.C. Xu, A new class of Zienkiewicz-type non-conforming element in any dimensions, Numer. Math. 106 (2007), 335-347.

46. L.B. Zhang, A parallel algorithm for adaptive local refinement of tetrahedral meshes using bisection, Numer. Math. Theor. Meth. Appl. 2 (2009), 65-89.

47. S.Y. Zhang, A family of $3 d$ continuously differentiable finite elements on tetrahedral grids, Appl. Numer. Math. 59 (2009), 219-233. 
48. O.C. Zienkiewicz and R.L. Taylor, The Finite Element Method for Solid and Structural Mechanics, 6th ed., Elsevier (Singapore), Pte Ltd., 2009.

Department of Mathematics, Sichuan Normal University, Chengdu 610066, China, EMAIL: LHL@SICNU.EDU.CN

LSEC, Institute of Computational Mathematics and Scientific/Engineering Computing, AMSS, Chinese Academy of Sciences, No. 55, East Road Zhong-Guan-Cun, Beijing 100190, China, and School of Mathematical Sciences, University of Chinese Academy of Sciences, Beijing 100049, China, Email: MPB@LSEC.CC.AC.CN

BeiJing 101 Middle School, EMAIL: Why@LSEC.CC.AC.CN 